\title{
Observational studies of Cepheid amplitudes
}

\section{Metallicity dependence of pulsation amplitudes}

\author{
L. Szabados and P. Klagyivik
}

Konkoly Observatory, Konkoly Thege út 15-17, 1121 Budapest XII, Hungary
e-mail: szabados@konkoly.hu

Received 3 August 2011 / Accepted 24 November 2011

\begin{abstract}
Context. Physical and phenomenological properties (radius, luminosity, shape of the light curve, etc.) of Cepheids strongly depend on the pulsation period, with the exception of the pulsation amplitude. A possible factor causing a wide range of pulsation amplitudes might be the different atmospheric metallicities of individual Cepheids.

Aims. We studied the influence exerted by the atmospheric iron content, $[\mathrm{Fe} / \mathrm{H}]$, on the pulsational amplitude of Galactic Cepheids. Methods. We searched for correlations between the $[\mathrm{Fe} / \mathrm{H}]$ value and both the observed amplitudes and amplitude related parameters. Results. The amplitude of the Cepheid pulsation slightly decreases with increasing iron abundance. This effect is more pronounced for the radial velocity variations and for the shorter pulsation periods. The wavelength dependence of photometric amplitudes is also found to be sensitive to the metallicity. Some of these effects are not consequences of differential line blanketing. Based on the calibrations of the metallicity sensitivity relationships, we derived photometric iron abundance for 21 Galactic Cepheids. The dichotomic behaviour dividing Galactic Cepheids that pulsate in the fundamental mode into short- and long-period groups at the period of 10.47 can be noticed in some diagrams that show metallicity-related dependences.

Conclusions. We confirm that variety in atmospheric metallicity in Cepheids contributes to the finite range of pulsation amplitudes at a given period. Effects of metallicity on the amplitudes revealed from observational data and the occurrence of the dichotomy also derived from phenomenological data have to be confirmed by appropriate theoretical models of stellar structure and pulsation.
\end{abstract}

Key words. stars: oscillations - stars: abundances - stars: variables: Cepheids - stars: fundamental parameters

\section{Introduction}

Classical Cepheids are radially pulsating supergiant stars. This type of pulsation, maintained by the $\kappa$-mechanism in the outer layers of the star, is stable in a narrow range of the effective temperature, in the nearly vertical instability strip in the Hertzsprung-Russell diagram. The fact that this pulsation is a free radial oscillation of the star results in a relationship between the period, $P$, and luminosity, $L$, of Cepheids.

Owing to the existence of the period-luminosity $(P-L)$ relationship, Cepheids are primary distance indicators in astronomy. The precise calibration of this relationship has been in the forefront of Cepheid-related studies for decades. A recently emerged aspect of these studies is the role of metallicity in shaping the $P-L$ relationship. In spite of thorough theoretical and observational investigations, the results on the metallicity dependence are controversial (see summaries by Romaniello et al. 2008; Marconi 2009; Freedman \& Madore 2010).

Along with other Cepheid-related relationships, the $P-L$ relationship is roughly linear if plotted against $\log P$. The only exception is the period-amplitude $(P-A)$ relationship. The wide range of possible photometric and radial velocity amplitudes at a given pulsation period may be caused by various effects (pulsation mode and energy, companion star(s), metallicity, etc.).

Motivated by the fact that the dependence of the pulsation amplitude on $\log P$ is neither linear nor unique, we studied the $P-A$ relationship of Galactic Cepheids in Paper I
(Klagyivik \& Szabados 2009). We revised the $P-A$ graphs for the $U, B, V, R_{\mathrm{C}}$, and $I_{\mathrm{C}}$ photometric bands. In addition, a $P-A$ graph was constructed using the observed peak-to-peak amplitudes of the pulsational radial velocity variations updating the much earlier diagrams (including that by Carson \& Stothers 1984 , to which we inadvertently missed to refer in Paper I). One purpose of constructing new $P-A$ diagrams has been to study the possible effect of metallicity on the pulsational amplitude, which may contribute to the observed finite range of actual amplitudes at a given pulsation period.

An influence of the chemical composition on the amplitude of pulsation is expected based on different patterns of the $P-A$ plots for Cepheids in our Galaxy and the Magellanic Clouds. van Genderen (1978) was the first who attributed the differences in the largest pulsation amplitudes of Cepheids to the different average metallicities of the respective host galaxies. If atmospheric metallicity has an influence on largest possible amplitude at a given pulsation period, then it should exert influence on the actual amplitude of individual Cepheids.

Here we study the effect of the atmospheric iron content, $[\mathrm{Fe} / \mathrm{H}]$, on peak-to-peak amplitudes and amplitude-related parameters introduced in Paper I for classical Cepheids of our Galaxy. Input values have been taken from Paper I (where the sources of the amplitude and spectroscopic $[\mathrm{Fe} / \mathrm{H}]$ data as well as the method of their homogenization is also described); in addition, $[\mathrm{Fe} / \mathrm{H}]$ values published recently by Luck \& Lambert (2011) and Luck et al. (2011) have also been taken into account 
Table 1. Average $[\mathrm{Fe} / \mathrm{H}]$ values for various groups of Cepheids.

\begin{tabular}{|c|c|c|c|c|c|c|c|c|c|}
\hline \multirow[t]{2}{*}{ Sample } & \multicolumn{3}{|c|}{ Solitary Cepheids } & \multicolumn{3}{|c|}{ Binary Cepheids } & \multicolumn{3}{|c|}{ Total sample } \\
\hline & {$[\mathrm{Fe} / \mathrm{H}]$} & $\sigma$ & $N$ & {$[\mathrm{Fe} / \mathrm{H}]$} & $\sigma$ & $N$ & {$[\mathrm{Fe} / \mathrm{H}]$} & $\sigma$ & $N$ \\
\hline Fundamental mode (F) Cepheids, $\log P>1.02$ & 0.167 & 0.164 & 49 & 0.184 & 0.154 & 34 & 0.174 & 0.160 & 83 \\
\hline Fundamental mode (F) Cepheids, $\log P<1.02$ & 0.091 & 0.158 & 114 & 0.103 & 0.100 & 78 & 0.096 & 0.137 & 192 \\
\hline First overtone (1OT) Cepheids & 0.108 & 0.142 & 28 & 0.068 & 0.118 & 24 & 0.089 & 0.133 & 52 \\
\hline
\end{tabular}

(after applying the same correction as for the $[\mathrm{Fe} / \mathrm{H}]$ data tabulated in Paper I). The present sample consists of 329 Galactic Cepheids with known spectroscopic $[\mathrm{Fe} / \mathrm{H}]$ value.

This paper is organized as follows. Section 2 deals with the period dependence of the iron content of Galactic Cepheids. Correlations between the $[\mathrm{Fe} / \mathrm{H}]$ value and various amplitudes and amplitude-related parameters are presented in Sect. 3. Consequences of our results are discussed in Sect. 4, while the conclusions are drawn in Sect. 5.

\section{Period dependence of $[\mathrm{Fe} / \mathrm{H}]$}

\subsection{Period $-[\mathrm{Fe} / \mathrm{H}]$ relationship}

Long-period Cepheids, which are more luminous (thus more massive) than their shorter period siblings, evolve more rapidly. As a consequence, longer period Cepheids are also younger, which is expressed by the period-age relationship (see, e.g., Efremov 2003). Owing to the continuous enrichment of the interstellar matter with heavy elements, the metal abundance of the younger stellar population is higher.

Figure 1 shows the $[\mathrm{Fe} / \mathrm{H}]$ value as a function of $\log P$. In all figures throughout this paper, Cepheids pulsating in the fundamental (F) mode are denoted by (blue) circles if $P<10^{\mathrm{d}} .47$ and (red) squares for $P>10 \mathrm{~d} 47$, while (green) triangles correspond to first overtone (1OT) pulsators. The replacement of the conventional period limit $(10 \mathrm{~d})$ separating short- and longperiod Cepheids by 10.47 was justified in Paper I. Empty and filled symbols are used for distinguishing Cepheids with and without known companions, respectively. In most of the subsequent figures, however, Cepheids with known companion(s) were disregarded because companion stars may falsify amplitudes. According to various pieces of evidence (see http:// www. konkoly.hu/CEP/intro.html), 137 Cepheids (of 329) have companion(s). This high frequency of occurrence of binaries (or multiple systems) in this Cepheid sample agrees with the statistics presented by Szabados (2003).

As expected, the average $[\mathrm{Fe} / \mathrm{H}]$ value slightly increases towards longer pulsation periods in Fig. 1, although there is a wide range of observed iron abundances at any period. This last observation is a consequence of the dependence of the chemical composition of stars on Galactic position (see Sect. 2.3).

The average value of the iron abundance of separate groups of Cepheids (F mode, short and long period; 1OT) is summarized in Table 1, separately for "solitary" Cepheids (i.e., for Cepheids without known companions), Cepheids with companions, and the whole sample.

The period dependence of the iron abundance was determined by the least-squares method. The line expressing the increase in the iron abundance with the pulsation period is

$[\mathrm{Fe} / \mathrm{H}]=-(0.012 \pm 0.027)+(0.149 \pm 0.030) \times \log P$

based on all 329 Cepheids in the sample. Freedman \& Madore (2011) could not find any period dependence of $[\mathrm{Fe} / \mathrm{H}]$ values for a sample of 22 Cepheids in the Large Magellanic Cloud. This

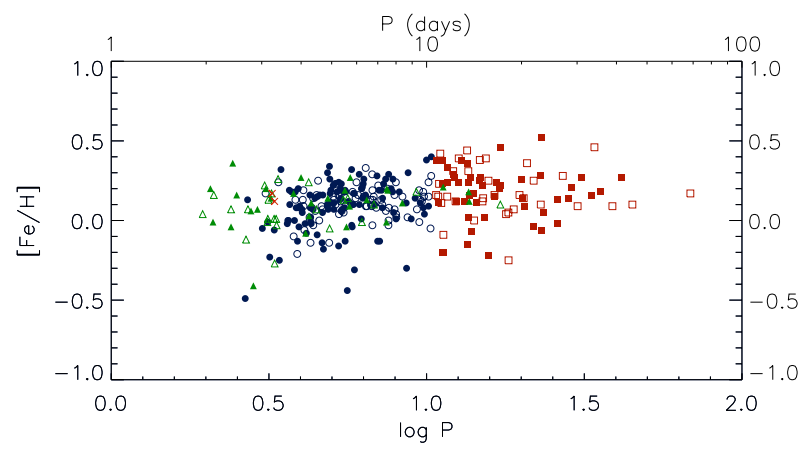

Fig. 1. Dependence of $[\mathrm{Fe} / \mathrm{H}]$ on the pulsation period. Circles represent F-mode Cepheids pulsating with short period $\left(P<10^{\mathrm{d}} .47\right)$, squares denote their long-period counterparts $(P>10 \mathrm{~d} 47)$, while triangles correspond to 1OT pulsators. Empty and filled symbols are used for distinguishing Cepheids with and without known companions, respectively.

contradicting result can be explained by the lack of short-period Cepheids in their sample.

This dependence is robust, it is not an artefact caused by outliers (i.e., most metal deficient) Cepheids. Omitting the six most deviating $[\mathrm{Fe} / \mathrm{H}]$ values $([\mathrm{Fe} / \mathrm{H}]<-0.25)$, the fit becomes

$[\mathrm{Fe} / \mathrm{H}]=(0.017 \pm 0.023)+(0.125 \pm 0.027) \times \log P$.

\subsection{Cepheid desert in the 8-10 day period interval}

The $P-A$ graphs show a prominent dip in the pulsation amplitude at $\log P=0.98$ for Galactic Cepheids (Paper I). At slightly shorter pulsation periods, there is a deficiency of Cepheids in the interval of $0.930<\log P<0.965$. This "zone of avoidance" was first mentioned by van Genderen (1970). A similar deficit is present in the period distribution of Cepheids in M 31 but no such feature appears in the $P-A$ plots for Magellanic Cepheids.

Nonlinear pulsation calculations were able to successfully reproduce this behaviour in the observed number distribution. Buchler et al. (1997) found that the fundamental mode limit cycles are unstable in the period domain 8-10 d for Cepheids with metallicity in the range $Z=0.014-0.035$. The corresponding stars pulsate in the first overtone with a period of $P_{1} \approx 0.7 P_{0}$, which causes a relative excess of 1 OT Cepheids in the $5.6-7.0$ period interval. This concerns our Galaxy and M 31, as well. Magellanic Clouds are of lower metallicity, therefore F-mode Cepheid pulsation is maintained in them in the period interval of $8-10 \mathrm{~d}$. According to this theoretical prediction, Galactic Cepheids within the period interval of 8-10 d may contain a much greater or much lesser amount of heavy elements in their atmosphere than the Galactic average.

Using the transformation formula between the heavy element abundance, $Z$, and the iron content, $[\mathrm{Fe} / \mathrm{H}]$, given by Caputo et al. (2001)

$\log Z=[\mathrm{Fe} / \mathrm{H}]-1.7$, 


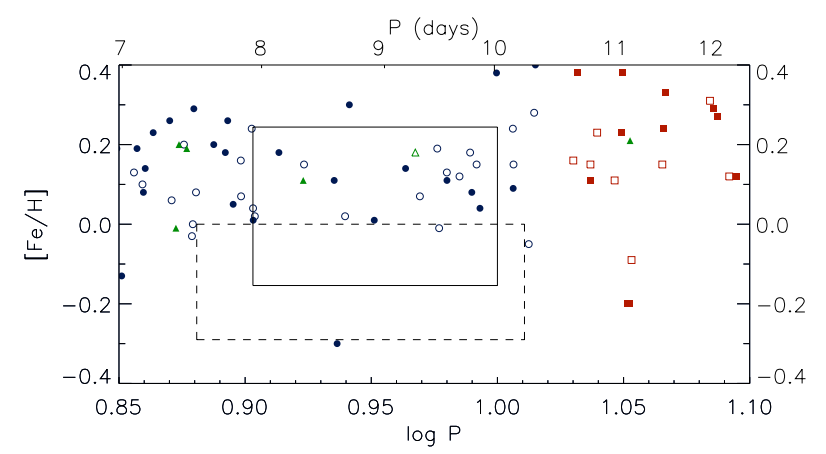

Fig. 2. Distribution of $[\mathrm{Fe} / \mathrm{H}]$ values near the "zone of avoidance". Cepheids with known companions are also plotted. The meaning of the symbols is the same as in Fig. 1. The theoretically predicted region of the Galactic Cepheid desert is represented by the solid line box, while the observed desert region is marked as the dashed line box.

the $0.014<Z<0.035$ interval of metallicity corresponds to a $-0.154<[\mathrm{Fe} / \mathrm{H}]<0.244$ range of iron abundance.

The relevant part of Fig. 1 is shown separately in Fig. 2. The box drawn with a solid line represents the theoretically obtained "zone of avoidance" as calculated by Buchler et al. (1997), while the borders of the observed Galactic Cepheid desert are marked with the box drawn with a dashed line. Our sample shows a deficiency of the F-mode Cepheids in the $0.88<\log P<1.01$ interval for the metallicity range of $0.00>[\mathrm{Fe} / \mathrm{H}]>-0.29$.

The region of the theoretical box only partially overlaps with the actual desert region of Cepheids that pulsate in the F-mode. The observed limits of the "zone of avoidance" imply a wider period interval in both directions and suggest that the mechanism computed by Buchler et al. (1997) is only valid for the $Z=0.010-0.020$ range. A revision of the theoretical picture is suggested.

\subsection{Galactic distribution of Cepheid metallicities}

The metal abundance of stars depends on the location in the host galaxy. The dependence of $[\mathrm{Fe} / \mathrm{H}]$ on galactic longitude in Cepheids has been studied by Andrievsky et al. (2004) - who also discussed the radial metallicity gradient observed in our Galaxy - and by Klagyivik \& Szabados (2007). Here we only study the distribution of $[\mathrm{Fe} / \mathrm{H}]$ values in the direction perpendicular to the Galactic plane. The distance from the Galactic plane was calculated for each Cepheid from the galactic latitude and the distance (taken from the Cepheid data base maintained at the David Dunlap Observatory - see Fernie et al. 1995). The results are shown in Fig. 3. Here and in the subsequent figures Cepheids with known companions have been disregarded.

The diagram shows a trend that Cepheids more distant from the Galactic plane are more deficient in iron. In this case, however, we are not facing an age effect. Classical Cepheids belong to the young stellar population, therefore the pattern seen in Fig. 3 does not imply a dependence of $[\mathrm{Fe} / \mathrm{H}]$ on the distance from the Galactic plane for the sample Cepheids. Instead, it can be interpreted as an artefact of merging Cepheids of all galactic longitudes in a single diagram. Most metal-poor Cepheids are situated in the outer spiral arm (constellations Aur, Cam, Cas, Per) where the disk extends farther perpendicularly to the Galactic plane than at shorter galactocentric distances (Binney $\&$ Merrifield 1998). The warp of the Galactic plane also contributes to the pattern seen in Fig. 3. From our viewpoint, the Galactic disk turns up to the north in the direction of Cygnus at

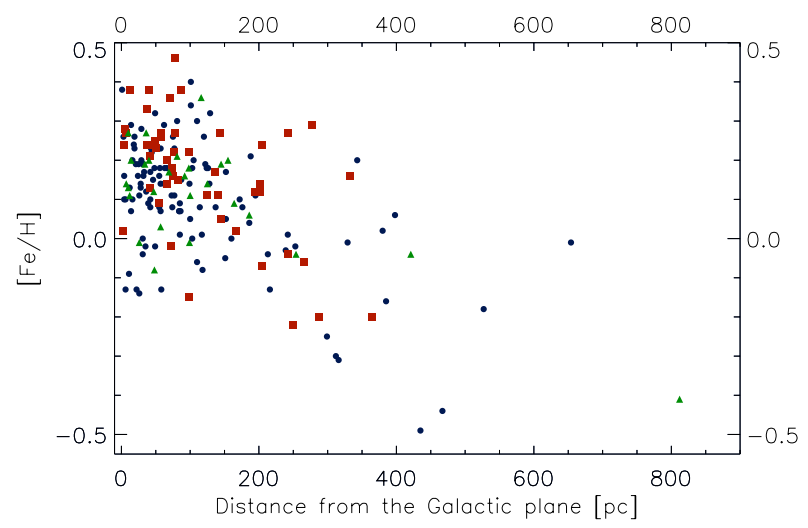

Fig. 3. Distribution of $[\mathrm{Fe} / \mathrm{H}]$ as a function of the distance from the Galactic plane. The meaning of the symbols is the same as in Fig. 1.

$\ell \simeq 90^{\circ}$, and south in the direction of Vela at $\ell \simeq 270^{\circ}$ (Perryman 2009).

\section{Effects of the iron content on the pulsation}

The pulsation amplitude of the Cepheids depends on several factors (period, presence of companions, mode and energy of pulsation - these latter two factors are closely related to the location of the star within the instability region), therefore it is not easy to distinguish the effect of metallicity.

\section{1. $[\mathrm{Fe} / \mathrm{H}]$ dependence of photometric amplitudes}

It is a realistic assumption that metallicity has an influence on the amplitude of pulsation. Using the upper envelope of the $P-A$ relationship derived in Paper I, no reasonable correlation could be revealed between the "amplitude defect" (at a given period), $\Delta A=A_{\lambda_{\max }}-A_{\lambda}$ and the iron content in any photometric band, nor for the radial velocity amplitudes.

It turned out, however, that the amplitudes themselves show a slight metallicity dependence if the Cepheids are properly grouped. Our findings are visualised in Fig. 4 for the photometric $B$ (upper panel), photometric $R_{\mathrm{C}}$ (middle panel), and the radial velocity amplitudes (lower panel). The metallicity effect is conspicuous for Cepheids pulsating in the 1OT and for short-period F-Cepheids, in the sense that more metal-deficient Cepheids pulsate with a larger amplitude. The correlation is most pronounced between the radial velocity amplitude and the iron content. There are several deviating points among long-period Cepheids: the low amplitude of the longest period Cepheids ( $\ell$ Car, RY Vel) is a known empirical fact (see Paper I) and some F-Cepheids whose period is near the value of the dichotomy (e.g., VX Per) mimic the behaviour of Cepheids with periods shorter than the limit of 10.47 ).

Table 2 summarizes the results of linear least-squares fits. Instead of the widely used ordinary least-squares regression of the dependent variable $y$ against the independent variable $x$, or OLS $(y \mid x)$, we used the OLS bisector method (Isobe et al. 1990) in these cases and all subsequent fits in this paper. This regression line treats the variables symmetrically instead of the $\operatorname{OLS}(y \mid x)$, which minimizes the sum of the squares of the $y$ residuals. The coefficients $a$ and $b$ in Table 2 correspond to the following formula:

$A_{X}=a+b \times[\mathrm{Fe} / \mathrm{H}]$ 
Table 2. Coefficients of the linear fit to the amplitude versus $[\mathrm{Fe} / \mathrm{H}]$ relationships (Eq. (4)) based on solitary Cepheids.

\begin{tabular}{|c|c|c|c|c|c|c|c|c|c|c|c|c|}
\hline \multirow[b]{2}{*}{ Sample } & \multicolumn{4}{|c|}{$A_{V_{\mathrm{RAD}}}$} & \multicolumn{4}{|c|}{$A_{B}$} & \multicolumn{4}{|c|}{$A_{R}$} \\
\hline & $\begin{array}{c}a \\
\left(\sigma_{a}\right)\end{array}$ & $\begin{array}{c}b \\
\left(\sigma_{b}\right)\end{array}$ & $N$ & $r$ & $\begin{array}{c}a \\
\left(\sigma_{a}\right)\end{array}$ & $\begin{array}{c}b \\
\left(\sigma_{b}\right)\end{array}$ & $N$ & $r$ & $\begin{array}{c}a \\
\left(\sigma_{a}\right)\end{array}$ & $\begin{array}{c}b \\
\left(\sigma_{b}\right)\end{array}$ & $N$ & $r$ \\
\hline F Cepheids, $\log P>1.02$ & $\begin{array}{l}53.39 \\
(0.85)\end{array}$ & $\begin{array}{l}-17.57 \\
(7.74)\end{array}$ & 31 & -0.239 & $\begin{array}{c}1.620 \\
(0.066)\end{array}$ & $\begin{array}{l}-0.254 \\
(0.255)\end{array}$ & 44 & -0.154 & $\begin{array}{c}0.817 \\
(0.029)\end{array}$ & $\begin{array}{l}-0.009 \\
(0.114)\end{array}$ & 46 & -0.011 \\
\hline F Cepheids, $\log P<1.02$ & $\begin{array}{l}37.01 \\
(2.36)\end{array}$ & $\begin{array}{l}-24.58 \\
(5.47)\end{array}$ & 58 & -0.515 & $\begin{array}{l}1.054 \\
(0.021)\end{array}$ & $\begin{array}{l}-0.273 \\
(0.110)\end{array}$ & 105 & -0.237 & $\begin{array}{c}0.571 \\
(0.012)\end{array}$ & $\begin{array}{l}-0.162 \\
(0.063)\end{array}$ & 108 & -0.239 \\
\hline 1OT Cepheids & $\begin{array}{l}17.55 \\
(1.59)\end{array}$ & $\begin{array}{c}-13.00 \\
(7.87)\end{array}$ & 24 & -0.340 & $\begin{array}{c}0.500 \\
(0.022)\end{array}$ & $\begin{array}{l}-0.339 \\
(0.127)\end{array}$ & 28 & -0.398 & $\begin{array}{c}0.277 \\
(0.015)\end{array}$ & $\begin{array}{l}-0.170 \\
(0.078)\end{array}$ & 27 & -0.347 \\
\hline
\end{tabular}

Notes. $N$ is the number of Cepheids in the given sample, $r$ is the correlation coefficient.
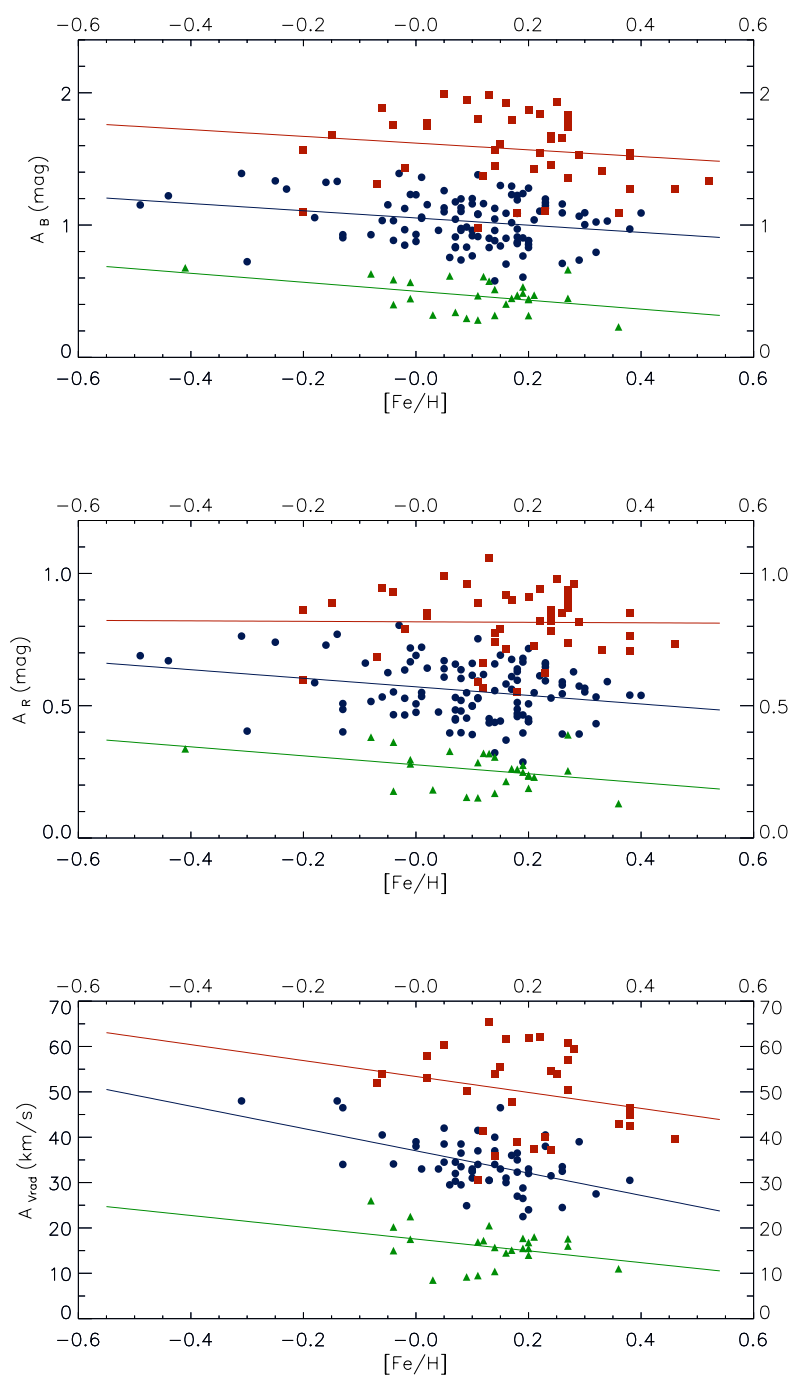

Fig. 4. Dependence of photometric $B$ (upper panel), $R_{\mathrm{C}}$ (middle panel), and radial velocity amplitudes (lower panel) on the atmospheric iron content. The meaning of the symbols is the same as in Fig. 1. The linear least-squares fit to each subsample is also plotted.

where the subscript $X$ refers to the relevant band. ER Aur has been omitted from the figures and the fits because its photometric amplitude turned out to be indefinite on closer inspection.

Figure 4 also confirms that the dichotomy between shortand long-period Cepheids occurs at $P=10 \mathrm{~d} 47$. Dividing fundamental-mode Cepheids into two groups at the conventional value of $P=10 \mathrm{~d} 0$, plots corresponding to Fig. 4 resulted in a less clear picture with more outliers.

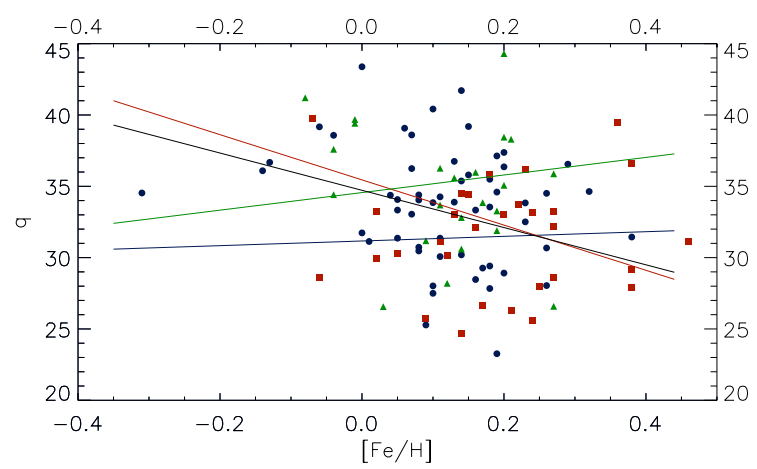

Fig. 5. Metallicity dependence of the $q$ amplitude ratio. The various fits are explained in the text.

Table 3. Coefficients of the linear fit to the $q$ versus $[\mathrm{Fe} / \mathrm{H}]$ relationship (Eq. (5)) based on solitary Cepheids.

\begin{tabular}{lcccccc}
\hline \hline Sample & $a$ & $\sigma_{a}$ & $b$ & $\sigma_{b}$ & $N$ & $r$ \\
\hline F Cepheids, $\log P>1.02$ & 31.17 & 1.97 & -15.85 & 6.05 & 30 & 0.017 \\
F Cepheids, $\log P<1.02$ & 35.45 & 0.88 & 1.63 & 9.34 & 56 & -0.236 \\
All F mode Cepheids & 34.72 & 0.89 & -13.06 & 5.51 & 86 & -0.204 \\
1OT Cepheids & 34.56 & 3.15 & 6.16 & 25.27 & 24 & 0.065 \\
\hline
\end{tabular}

\subsection{Dependence of amplitude parameters on $[\mathrm{Fe} / \mathrm{H}]$}

The importance of the amplitude ratio $A_{V_{\mathrm{RAD}}} / A_{B}$ (referred to as the $q$ parameter) was discussed in Paper I. It is sensitive to both the oscillation mode and the presence of a companion to the Cepheid. Unfortunately, the two effects cannot be separated from each other, which diminishes the diagnostic value of the $q$ parameter. An additional complication is that $q$ also depends on the metallicity of the pulsating star, as shown below.

The relationship between $q$ and $[\mathrm{Fe} / \mathrm{H}]$ is shown in Fig. 5, involving only solitary Cepheids. The meaning of the symbols is the same as in Fig. 1. Linear least-squares fits have been applied to each group separately, and the results are summarized in Table 3. Coefficients $a$ and $b$ correspond to the formula

$q=a+b \times[\mathrm{Fe} / \mathrm{H}]$.

The last two columns of Table 3 give the number of data points, $N$, involved in the linear regression analysis and the correlation coefficient, $r$. The fits are also shown in Fig. 5: the red linear section is for the long-period $\mathrm{F}$ pulsators, the blue section for their short-period counterparts, the black line denotes the fit to the sample of all F Cepheids, while the green section represents the fit for the 1OT Cepheids.

In Paper I we quantified the wavelength dependence of the photometric amplitudes with two numerical parameters. Plotting 

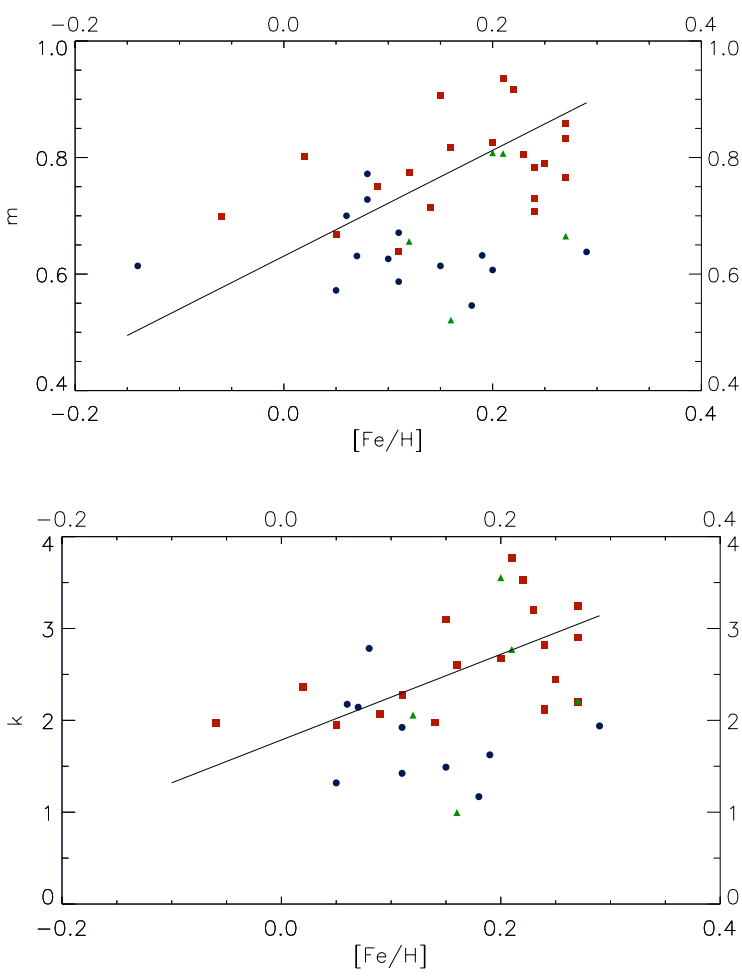

Fig. 6. Metallicity dependence of the parameters $m$ (upper panel) and $k$ (lower panel). The meaning of the symbols is the same as for Fig. 1. The linear least-squares fit to the sample of long-period Cepheids is also shown.

the amplitude of the light variations measured in the $U, B, V$, and $R_{\mathrm{C}}$ bands normalized to the amplitude observed in $B$ band against $1 / \lambda$, the distribution of the points is roughly linear for a given Cepheid (Fernie 1979). The slope of the straight line fitted to these points has been defined as the $m$ parameter for individual stars (Paper I).

The top panel of Fig. 6 shows the relation between the iron content and the $m$ parameter for F-mode solitary Cepheids. (Data for four 1OT Cepheids are also plotted.) In the case of longperiod Cepheids, metallicity has a noticeable effect. The higher the $[\mathrm{Fe} / \mathrm{H}]$, the larger the $m$ parameter, i.e., the amplitude decreases stronger with increasing wavelength. The linear fit to the points representing 20 Cepheids with $P>10.47$ (also shown in the upper panel of Fig. 6) is

$m=0.631( \pm 0.033)+0.908( \pm 0.142) \times[\mathrm{Fe} / \mathrm{H}]$.

The correlation coefficient of the linear regression is $r=0.416$.

The other parameter, $k$, characterizes the wavelength dependence of photometric amplitudes in five $\left(U, B, V, R_{\mathrm{C}}\right.$, and $\left.I_{\mathrm{C}}\right)$ photometric bands. Approximating the amplitude-wavelength graph with an exponential curve of the form

(Amplitude $)_{\lambda}=c_{1}+c_{2} \times \lambda^{-k}$

serves as the definition of the $k$ parameter introduced in Paper I.

The dependence of the $k$ parameter on the atmospheric iron content is shown in the bottom panel of Fig. 6. The linear section shown there corresponds to the linear least-squares fit to the data of 19 long-period $(P>10.47)$ Cepheids:

$k=1.786( \pm 0.148)+4.665( \pm 1.010) \times[\mathrm{Fe} / \mathrm{H}]$.

The correlation coefficient is $r=0.470$.
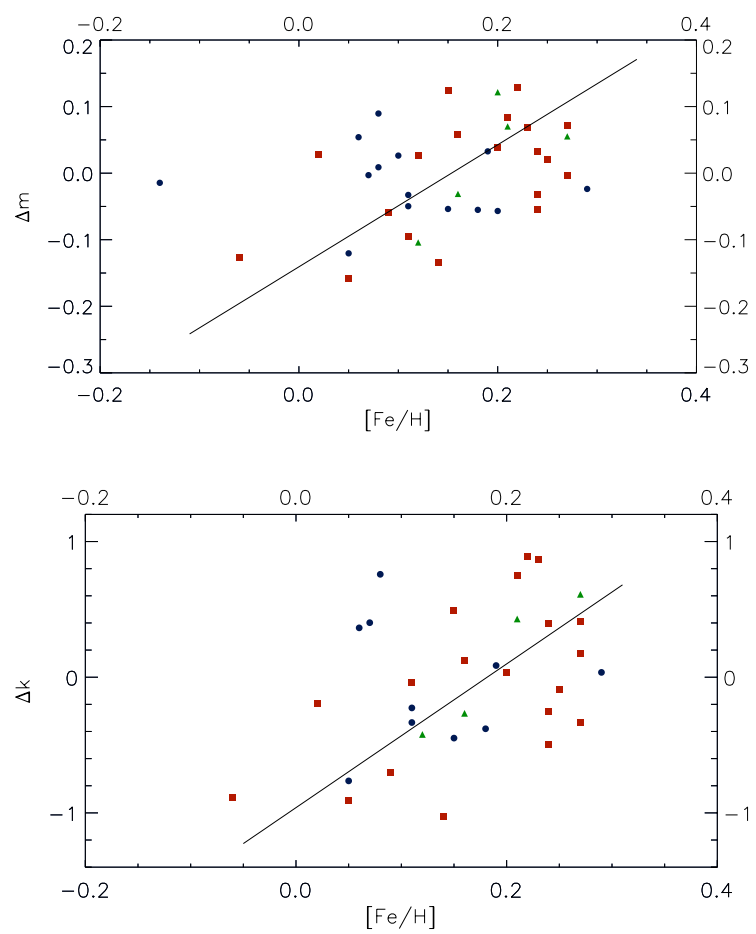

Fig. 7. Metallicity dependence of $\Delta m$ and $\Delta k$ parameters and the linear least-squares fit for the solitary, long-period Cepheids. The meaning of the symbols is the same as for Fig. 1.

The effect of metallicity on the wavelength dependence of the pulsation amplitude of the long-period Cepheids is again clearly seen.

Both $m$ and $k$ parameters depend on the pulsation period, as well (Paper I). To quantify the real $[\mathrm{Fe} / \mathrm{H}]$ dependence of these parameters, the period dependence has to be removed. Let $\Delta m$ denote the difference between the actual $m$ value of a Cepheid and the mean $m$ calculated for the given pulsation period from Eq. (12) in Paper I. Likewise, $\Delta k$ is introduced for characterizing the deviation of $k$ values of individual Cepheids from the ridge line given by Eq. (14) in Paper I.

The $\Delta m$ and $\Delta k$ values as a function of $[\mathrm{Fe} / \mathrm{H}]$ are plotted in Fig. 7. Linear least-squares fits to the data for the long-period Cepheids resulted in the following equations:

$\Delta m=-0.150( \pm 0.032)+0.923( \pm 0.143) \times[\mathrm{Fe} / \mathrm{H}]$

based on 20 data points $(r=0.500)$ and

$\Delta k=-0.961( \pm 0.193)+5.297( \pm 1.017) \times[\mathrm{Fe} / \mathrm{H}]$

from 19 data points $(r=0.540)$. The subsamples of short-period Cepheids and s-Cepheids (though they are plotted in Fig. 7) span a rather narrow metallicity interval and include too few stars to obtain meaningful results of this type.

Figure 7 shows that both $\Delta m$ and $\Delta k$ increase with increasing iron abundance and the scatter is narrower than for the plots in Fig. 6.

\section{3. $[\mathrm{Fe} / \mathrm{H}]$ values derived from the amplitudes}

The strength of the metallic lines in stellar spectra has an influence on the brightness observable in particular in blue and violet photometric bands. For intermediate-band photometries, even calibrations exist between intrinsic (i.e., reddening corrected) colour indices and the $[\mathrm{Fe} / \mathrm{H}]$ value. Eggen (1985) determined the metallicity of about a hundred Cepheids observed in 
the Strömgren system. The broad-band Washington system has been elaborated specifically for determining atmospheric abundances of stars whose temperature is similar to that of Cepheids (Canterna 1976). Metal abundances derived from photometric data obtained in this system are uncertain by a factor of two. Colour indices of the broad-band Johnson system (in which the overwhelming majority of Cepheid photometries has been carried out) are not ideal for abundance studies, but colour indices of this $U B V$ system were also calibrated in terms of $[\mathrm{Fe} / \mathrm{H}]$ via the line blanketing effect (see Karaali et al. 2011, and references therein). The drawback of the applications of these calibrations to photometric data on Cepheids is the uncertainty in the colour excess of individual Cepheids.

The metallicity dependence of the amplitude parameters discussed in Sect. 3.2 can be used for determining the $[\mathrm{Fe} / \mathrm{H}]$ of individual long-period Cepheids, based in Eqs. (9) and (10), if peak-to-peak photometric amplitudes are known, and Eq. (5), if the amplitude of the pulsational radial velocity variation and the photometric $B$ amplitude are known. We refer to these $[\mathrm{Fe} / \mathrm{H}]$ values as photometric iron abundances.

Photometric and spectroscopic $[\mathrm{Fe} / \mathrm{H}]$ values are compared in Fig. 8 using those "solitary" Cepheids for which both kinds of iron abundance is known. The vertical axis shows $[\mathrm{Fe} / \mathrm{H}]_{\Delta m}$ (upper panel) as derived from the metallicity dependence of the $\Delta m$ parameter based in Eq. (9), the sample consists of 20 F Cepheids; $[\mathrm{Fe} / \mathrm{H}]_{\Delta k}$ (lower panel) as derived from the metallicity dependence of the $\Delta k$ parameter based in Eq. (10), the sample consists of 19 F Cepheids.

Figure 8 shows that from the $\Delta k$ and $\Delta m$ parameters one can determine an approximate $[\mathrm{Fe} / \mathrm{H}]$ value for long-period Cepheids with an uncertainty of \pm 0.15 dex. The newly determined photometric $[\mathrm{Fe} / \mathrm{H}]$ values for 21 long-period Cepheids are listed in Table 4, whose columns contain the following data: name of the Cepheid; pulsation period (rounded to 3 decimal figures); $[\mathrm{Fe} / \mathrm{H}]_{\mathrm{sp}}$, spectroscopic iron abundance taken from Table 1 in Paper I or from the more recent lists by Luck \& Lambert (2011) and Luck et al. (2011); $[\mathrm{Fe} / \mathrm{H}]_{\Delta m} ;[\mathrm{Fe} / \mathrm{H}]_{\Delta k}$, respectively.

For the long-period Cepheid GY Sge (0.22) this is the first metallicity estimation (with an estimated uncertainty of \pm 0.04 dex).

\section{Discussion}

According to our result, photometric amplitudes become larger with decreasing iron abundance. This is in contrast to the conclusion drawn by Paczyński \& Pindor (2000), who compared the photometric amplitudes of long-period Cepheids in the two Magellanic Clouds, and found that the higher the metal content of the host galaxy, the larger the amplitude. Their study was not based on the metallicity of individual Cepheids, instead, Paczyński \& Pindor compared the median amplitudes and assigned the mean metallicity of the host galaxy to the respective amplitude value. That method is an oversimplification because the metallicity of individual Cepheids is a function of the location within the host galaxy, and the sample investigated by Paczyński \& Pindor was too small (35 Cepheids in the SMC, 33 in the LMC, and 42 in the Milky Way Galaxy) to be representative for the whole galaxy. In addition, blending by unresolved companions might also bias the photometric amplitudes derived for Magellanic Cepheids.

An alternative explanation for the significant difference between the mean amplitudes of Cepheids in the three galactic systems can be a difference in the helium content in the stars
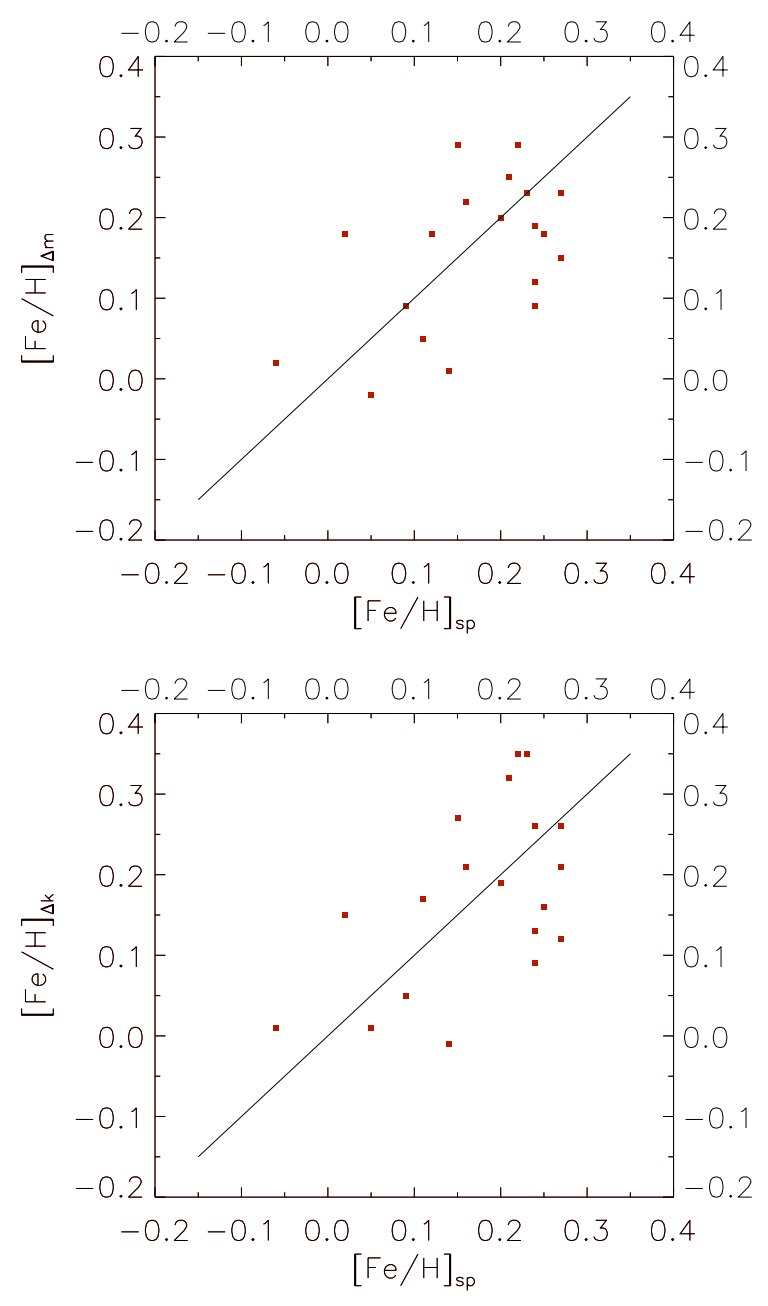

Fig. 8. Correlations between photometric and spectroscopic $[\mathrm{Fe} / \mathrm{H}]$ values. Spectroscopic $[\mathrm{Fe} / \mathrm{H}]$ is indicated along the abscissa. The various photometric iron abundances are explained in the text. The 1:1 correspondence is shown by a linear section in each panel.

whose observational verification is beyond the current spectroscopic capabilities. The location of the instability region in the Hertzsprung-Russell diagram also depends on the helium abundance (Becker et al. 1977; Marconi et al. 2005), and the differing effective temperature may also alter the pulsational properties of Cepheids.

Based on extensive spectroscopic investigations of classical Cepheids, Luck \& Andrievsky (2004), Andrievsky et al. (2006), Kovtyukh et al. (2006), and Luck et al. (2008) pointed out that there are no observable changes in the chemical abundance during the pulsational cycle. Variations in the effective temperature during the pulsation, however, result in variable strength of the metallic lines in spectra of Cepheids because line blanketing is stronger during the phases of lower temperature which in turn results in apparently larger photometric amplitudes for more metal-deficient Cepheids, especially in the shorter wavelength $B$ and $U$ bands. This effect is a plausible explanation for the observed dependence of the photometric amplitudes on the iron content shown in Fig. 4.

Differential line blanketing, however, does not offer an explanation for the correlations between the radial velocity amplitude and $[\mathrm{Fe} / \mathrm{H}]$ value (Fig. 4, lower panel). 
Table 4. The newly determined photometric iron abundances.

\begin{tabular}{lcccc}
\hline \hline Cepheid & $\begin{array}{c}P \\
(\mathrm{~d})\end{array}$ & {$[\mathrm{Fe} / \mathrm{H}]_{\mathrm{sp}}$} & {$[\mathrm{Fe} / \mathrm{H}]_{\Delta m}$} & {$[\mathrm{Fe} / \mathrm{H}]_{\Delta k}$} \\
\hline SZ Aql & 17.139 & 0.22 & 0.27 & 0.35 \\
TT Aql & 13.755 & 0.27 & 0.20 & 0.23 \\
XY Car & 12.439 & 0.12 & 0.15 & - \\
XZ Car & 16.650 & 0.24 & 0.05 & 0.01 \\
RW Cas & 14.790 & 0.27 & 0.11 & 0.05 \\
X Cyg & 16.386 & 0.15 & 0.26 & 0.25 \\
TX Cyg & 14.710 & 0.25 & 0.14 & 0.11 \\
CD Cyg & 17.076 & 0.20 & 0.16 & 0.14 \\
V609 Cyg & 31.080 & 0.27 & 0.11 & 0.18 \\
SV Mon & 15.233 & 0.02 & 0.15 & 0.09 \\
UU Mus & 11.636 & 0.24 & 0.08 & 0.07 \\
U Nor & 12.655 & 0.24 & 0.15 & 0.23 \\
VX Per & 10.889 & 0.11 & 0.01 & 0.12 \\
VZ Pup & 23.163 & -0.06 & -0.03 & -0.08 \\
BN Pup & 13.673 & 0.16 & 0.18 & 0.16 \\
GY Sge & 51.612 & - & 0.21 & 0.24 \\
KQ Sco & 28.699 & 0.21 & 0.22 & 0.31 \\
RY Vel & 28.134 & 0.14 & -0.04 & -0.11 \\
RZ Vel & 20.411 & 0.09 & 0.05 & -0.03 \\
SW Vel & 23.436 & 0.05 & -0.07 & -0.09 \\
DR Vel & 11.199 & 0.23 & 0.20 & 0.34 \\
\hline
\end{tabular}

Notes. See the explanation in the text.

The metallicity sensitivity of the wavelength dependence of the photometric amplitudes (Figs. 6, 7) can be a result of the temperature sensitivity of the differential line blanketing effect.

Metallicity is only one of the factors that has an influence on the pulsation amplitude. Another factor is the position of the Cepheid within the instability region. This aspect has been studied by Kanbur \& Ngeow in a series of papers. In their first paper (Kanbur \& Ngeow 2004) the dependence of the photometric $V$ amplitude on the $V-I$ colour index (corrected for reddening) was investigated, based on three galactic systems. The sample consisted of 154, 634, and 391 Cepheids in the Galaxy, LMC, and SMC, respectively. It is noteworthy that they found linear relationships between the $A_{V}$ and $(V-I)_{0}$ for Cepheids in each galactic sample, and the slope of the lines shows a systematics according to the average metallicity of the host galaxy. Obviously, therefore, a part of the scatter in Figs. 4-8 is caused by the colour (temperature) dependence of the pulsation amplitude. The separation of the effects of colour and metallicity on the pulsation amplitudes is left to a future study.

In addition to the amplitudes, metallicity is expected to have an influence on the shape of the light curve. These correlations exist for RR Lyrae stars (Kovács \& Zsoldos 1995; Jurcsik \& Kovács 1996). Because pulsation of Cepheids and RR Lyrae type stars is driven by the same mechanism, one expects that the light curve shape is sensitive to the atmospheric metal content for Cepheids, too. A study of the metallicity sensitivity of Fourier coefficients of the phase curves of Galactic Cepheids is in progress.

As an important by-product, we confirmed the separation of Galactic classical Cepheids into short- and long-period ones at the limiting pulsation period of $10 \mathrm{~d} .47$, especially by Fig. 4. It is noteworthy that the limiting period of $10 \mathrm{~d} 47$ is close to the resonance centre of $2 P_{2 \mathrm{OT}}=P_{\mathrm{F}}$ of the Cepheid pulsation (where $P_{\mathrm{F}}$ is the fundamental period, $P_{2 \mathrm{OT}}$ is the period of the second overtone). This resonance causes the famous Hertzsprung progression of the light curve shape of Cepheids. The value of the resonance centre is, however, not definite: from the observational point of view, the discontinuity of the Fourier phase parameters near $10 \mathrm{~d}$ as derived from the Cepheid light curves is not sharp at all (see Soszyński et al. 2008, 2010, for LMC and SMC Cepheids, respectively). From the point of view of theory, the resonance centre is model-dependent: models calculated by Klapp et al. (1985) predict the minimum amplitude for both resonant modes between 10.1 and $10 \mathrm{~d} .4$, quite close to the value of the dichotomy determined in Paper I. However, the cause of the different behaviour of short- and long-period Cepheids remains open. Simon \& Moffett (1985) assume that the long- and short-period Cepheids reach their limit cycles in different ways. Figure 4 is another manifestation of this phenomenon. The situation is, however, even more complicated because the resonance centre itself is a function of the metallicity of the modelled stars (Simon \& Kanbur 1994).

\section{Conclusion}

We confirmed that the observed variety in the atmospheric iron content of Cepheids contributes to the wide range of the actual pulsation amplitudes: more metal-deficient Cepheids tend to pulsate with larger amplitudes. We were able to reveal the metallicity dependence of several parameters $(q, k, m, \Delta k$, and $\Delta m)$ of Galactic Cepheids. In physical terms, these relationships mean that the amplitude of the radial velocity variations, the ratio of photometric to radial velocity amplitudes, and the wavelength dependence of the photometric amplitudes depend on the iron abundance of the pulsating stellar atmosphere. In some cases, the metallicity sensitivity of the amplitude parameters is not the consequence of the differential line blanketing with the result that stars of higher metallicity are fainter towards shorter wavelengths.

These correlations, although not strong, can still be used for deriving the $[\mathrm{Fe} / \mathrm{H}]$ value of individual Cepheids from photometric observational data. Improvement of these correlations is mainly hindered by the current uncertainties in the spectroscopic abundance determination: individual $[\mathrm{Fe} / \mathrm{H}]$ values have an error of $0.05-0.10$. From multicolour photometric data, we derived a photometric $[\mathrm{Fe} / \mathrm{H}]$ value for $21 \mathrm{Galactic}$ long-period Cepheids, one of which (GY Sge) is lacking prior spectroscopic abundance determination.

Availability of direct spectroscopic abundance values and multicolour light curves of Cepheids in the Magellanic Clouds would extend the newly found correlations to more negative $[\mathrm{Fe} / \mathrm{H}]$ values because the interval of atmospheric iron content of the Galactic Cepheids (typically $-0.25<[\mathrm{Fe} / \mathrm{H}]<+0.25$ ) is not particularly wide. Quite a few Cepheids more deficient in iron are known in the outer spiral arm of the Milky Way system but these Cepheids are so distant that their available spectroscopic $[\mathrm{Fe} / \mathrm{H}]$ values are too uncertain for calibration purposes. Magellanic Cepheids are much more metal-deficient: the mean $[\mathrm{Fe} / \mathrm{H}]$ is $-0.34 \pm 0.03$ for the $\mathrm{LMC}$ and $-0.64 \pm 0.04$ for the SMC (Keller \& Wood 2006). The available observational data on the abundance of individual Magellanic Cepheids are scanty: photometric metallicity values determined by Harris (1981) using the Washington system resulted in metallicities of limited accuracy, and spectroscopic metallicities for a sample consisting of 12-12 Cepheids in each cloud (Romaniello et al. 2005).

The finite interval of actual $[\mathrm{Fe} / \mathrm{H}]$ values of Cepheids situated in different parts of the same galaxy is a drawback when attempting to decrease the scatter of the $P-L$ relationship for Cepheids in individual galaxies and in settling the question of the metallicity sensitivity of the $P-L$ relationship. The wide 
variety of iron abundance values of Cepheids in the same host galaxy is a reason to be cautious when assigning an "average" metallicity to all Cepheids in a given galaxy. As a rule, the determination of the heavy element abundance of external galaxies (except the nearest ones) is based on the chemical properties of their bright HII regions (Zaritsky et al. 1994). Metallicity of these regions, however, corresponds to youngest population of stars involving long-period Cepheids. Short-period Cepheids are somewhat older (and evolve slower) and may have formed from a less processed interstellar matter.

Both the effect of metallicity (of non-blanketing origin) on the amplitudes revealed from observational data and the occurrence of the dichotomy at the limit of 10.47 (including its relation to the $2 P_{2 \mathrm{OT}}=P_{\mathrm{F}}$ resonance phenomenon) have to be investigated through appropriate theoretical models of stellar pulsation.

Acknowledgements. This research was supported by the European Space Agency (ESA) and the Hungarian Space Office via the PECS programme (contract No. 98090). The authors are indebted to Drs. Sz. Csizmadia, M. Kun, N. Nardetto, C.-C. Ngeow, and the referee for their remarks leading to a better presentation of the results.

\section{References}

Andrievsky, S. M., Luck, R. E., Martin, P., \& Lépine, J. R. D. 2004, A\&A, 413, 159

Andrievsky, S. M., Luck, R. E., \& Kovtyukh, V. V. 2006, AJ, 130, 1880

Becker, S. A., Iben, I., Jr., \& Tuggle, R. S. 1977, ApJ, 218, 633

Binney, J., \& Merrifield, M. 1998, Galactic Astronomy (Princeton University Press), Chapter 9

Buchler, J. R., Goupil, M. J., \& Piciullo, R. 1997, ApJ, 491, L99

Canterna, R. 1976, AJ, 81, 228

Caputo, F., Marconi, M., Musella, I., \& Pont, F. 2001, A\&A, 372, 544

Carson, T. R., \& Stothers, R. B. 1984, ApJ, 276, 593
Efremov, Yu. N. 2003, Astron. Rep., 47, 1000

Eggen, O. J. 1985, AJ, 90, 1278

Fernie, J. D. 1979, PASP, 91, 67

Fernie, J. D., Beattie, B., Evans, N. R., \& Seager, S. 1995, IBVS, 4148

Freedman, W. L., \& Madore, B. F. 2010, ARA\&A, 48, 673

Freedman, W. L., \& Madore, B. F. 2011, ApJ, 734, 46

Harris, H. C. 1981, AJ, 86, 1192

Isobe, T., Feigelson, E. D., Akritas, M. G., \& Babu, G. J. 1990, ApJ, 364, 104

Jurcsik, J., \& Kovács, G. 1996, A\&A, 312, 111

Kanbur, S. M., \& Ngeow, C.-C. 2004, MNRAS, 350, 962

Karaali, S., Bilir, S., Ak, S., Yaz, E., \& Coskunoglu, B. 2011, PASA, 28, 95

Keller, S. C., \& Wood, P. R. 2006, ApJ, 642, 834

Klagyivik, P., \& Szabados, L. 2007, Astron. Nachr., 328, 825

Klagyivik, P., \& Szabados, L. 2009, A\&A, 504, 959 (Paper I)

Klapp, J., Goupil, M. J., \& Buchler, J. R. 1985, ApJ, 296, 514

Kovács, G., \& Zsoldos, E. 1995, A\&A, 293, L57

Kovtyukh, V. V., Andrievsky, S. M., Belik, S. I., \& Luck, R. E. 2006, AJ, 129, 433

Luck, R. E., \& Andrievsky, S. M. 2004, AJ, 128, 343

Luck, R. E., \& Lambert, D. L. 2011, AJ, 142, 136

Luck, R. E., Andrievsky, S. M., Fokin, A., \& Kovtyukh, V. V. 2008, AJ, 136, 98

Luck, R. E., Andrievsky, S. M., Kovtyukh, V. V., Gieren, W. P., \& Graczyk, D. 2011, AJ, 142, 51

Marconi, M. 2009, Mem. Soc. Astron. Ital., 80, 141

Marconi, M., Musella, I., \& Fiorentino, G. 2005, ApJ, 632, 590

Paczyński, B., \& Pindor, B. 2000, ApJ, 533, L103

Perryman, M. 2009, Astronomical Applications of Astrometry (Cambridge Univ. Press), 536

Romaniello, M., Primas, F., Mottini, M., et al. 2005, A\&A, 429, L37

Romaniello, M., Primas, F., Mottini, M., et al. 2008, A\&A, 488, 731

Simon, N. R., \& Kanbur, S. M. 1994, ApJ, 429, 772

Simon, N. R., \& Moffett, T. J. 1985, PASP, 97, 1078

Soszyński, I., Poleski, R., Udalski, A., et al. 2008, Acta Astron., 58, 163

Soszyński, I., Poleski, R., Udalski, A., et al. 2010, Acta Astron., 60, 17

Szabados, L. 2003, Inf. Bull. Var. Stars, 5394

van Genderen, A. M. 1970, A\&A, 7, 244

van Genderen, A. M. 1978, A\&A, 65, 147

Zaritsky, D., Kennicutt, R. C. Jr., \& Huchra, J. P. 1994, ApJ, 420, 87 